Bol. Acad. peru. leng. 65. 2019 (11-31)

\title{
HACIA UNA PERIODIZACIÓN DE LA POESÍA PERUANA DEL SIGLO XX
}

\section{TOWARDS A PERIODIZATION OF THE PERUVIAN POETRY OF THE TWENTIETH CENTURY}

\author{
Luis Landa
}

\section{Resumen:}

El presente texto resume cuatro alcances de una investigación que devino en la tesis doctoral de 2018: "Asir el tiempo de la palabra: revisión de la periodización de la poesía peruana del siglo XX». De tal forma, se da cuenta del vacío en el estudio que se centra exclusivamente en la evolución de nuestra poesía. Como derivado de esta deficiente situación, la transmisión de la historia que se desarrolla desde la escuela hasta los estudios superiores expresa una mezcla de conceptos - como movimientos, corrientes, épocas y generaciones- que no grafican la naturaleza de la lírica. Por ello, en este artículo, se muestra la alternativa de la tesis de apostar por el concepto «periodo» que reorganice la percepción de la poesía peruana durante el siglo xx. Con un panorama de cuatro periodos, se reordenan las manifestaciones poéticas que surgieron desde los últimos años del siglo XIx, así como las que se vienen mostrando en estas primeras décadas del siglo xxi. Asimismo, se descarta la automática forma de apreciar la organización de poetas por décadas bajo el falso carácter de una generación artificial. 


\section{Abstract:}

The present text summarizes four scopes of an investigation that became the doctoral thesis of 2018: «Take the time of the word: review of the periodization of Peruvian poetry of the twentieth century.» In this way, he realizes the emptiness in the study that focuses exclusively on the evolution of our poetry. As a result of this poor situation, the transmission of history that develops from school to higher education expresses a mixture of concepts - such as movements, currents, times and generations - that do not graph the nature of the lyric. Therefore, this article shows the alternative of the thesis of betting on the concept «period» that reorganizes the perception of Peruvian poetry during the twentieth century. With an outlook of four periods, the poetic manifestations that emerged since the last years of the 19th century are reordered, as well as those that have been showing in these first decades of the 21st century. Likewise, the automatic way of appreciating the organization of poets for decades is ruled out under the false character of an artificial generation.

Palabras clave: periodización, periodo, generación, poesía peruana, canon.

Key words: periodization, period, generation, Peruvian poetry, canon.

Fecha de recepción:

Fecha de aceptación:
$29 / 03 / 2019$

$31 / 05 / 2019$

Generación del 50, generación del 60, generación del 70, generación del 80, generación del 90, generación del 2000 y igeneración del 2010 ? El evidente vacío en la reflexión sobre la poesía peruana contemporánea obliga a echar mano de un concepto como el de «generación» para ubicar a los autores y el efecto que concitan sus obras. Como se aprecia de manera notoria y algo autómata al comienzo de este texto, el problema de aplicar este concepto resulta en una serie artificial sin sentido profundo de caracterización, es decir, se trata de producir una 
https://doi.org/10.46744/bapl.201901.001

segmentación por décadas poco realista - porque desvirtúa el concepto de «generación»— y que se percibe ajena a la naturaleza del fenómeno literario mismo. Nos encontramos así en un entrampamiento del cual propongo salir a través de la aplicación de un viejo concepto válido y vigente: el "periodo». Estas ideas componen el cuerpo de mi tesis doctoral «Asir el tiempo de la palabra: revisión de la periodización de la poesía peruana del siglo xx» [http://cybertesis.unmsm.edu.pe/ handle/cybertesis/8365 show $=$ full] (2018). En este trabajo de investigación, doy cuenta de cuatro puntos fundamentales sobre los que se fundamenta la reflexión: la manipulación inadecuada del concepto «generación»; el caos en el régimen conceptual en la actual periodización; el vacío en la reflexión exclusiva sobre la evolución natural de nuestra poesía; la propuesta de una alternativa basada en el concepto de «periodo» que ilumine particularmente el desarrollo del fenómeno poético hasta la actualidad.

En primer lugar, en cuanto al abuso de "generación», deben considerarse, ante todo, los autores, cuyas teorías se encuentran transgredidas. Wilhelm Pinder (Elproblema de las generaciones en la historia del arte de Europa, 1924), Julius Petersen («Las generaciones literarias», 1930), —y, especialmente, en nuestro idioma- Pedro Salinas, Azorín, José Ortega y Gasset («En torno a Galileo», 1933) y Julián Marías (El método histórico de las generaciones, 1949) son algunos de los nombres más ilustres que han disertado al respecto. En diferentes textos, el filósofo español José Ortega y Gasset ha explicitado los requisitos para reconocer cuándo nos encontramos ante una generación. Basado en el biorritmo humano, Ortega y Gasset establece la duración aproximada de las generaciones en quince años y no en diez. Los cambios en la producción poética de los autores determinan la aparición o declive de una generación y, por ello, las coincidencias de nacimiento (misma edad y contacto vital) sirven para orientar claros horizontes o periodos de producción. Por lo tanto, la repartición por décadas no encuentra justificación, pues ni las cifras redondas son determinantes ni los diez años son suficientes para la emergencia y hegemonía de una generación de poetas en un esquema tantas veces repetido y artificioso. Mucho menos caben en una década los treinta años de gestación y de gestión 
que constituyen una generación. Es evidente la tergiversación del concepto propuesto por Ortega y Gasset si se consideran, además, sus propias palabras:

«A diferencia, en efecto, de todas las otras teorías sobre las generaciones y aun de la idea tradicional y viejísima acerca de ellas, yo las tomo, no como una sucesión, sino como una polémica, siempre que se entienda en serio esta palabra y no se la frivolice» (Ortega y Gasset, 2012: 509).

Aunque el pensador español pretendía esclarecer la diferencia entre las generaciones históricas y las generaciones genealógicas, lo cierto es que las fechas de nacimiento harían que un poeta como Roger Santiváñez (Piura, 1956), que ha trascendido tanto para la generación del 70 como para la del 80 y aun para la del 90, sea asumido como la excepción a la regular determinación generacional, en lugar del ejemplo coherente de lo que reúne un periodo: la injerencia de su producción. Asimismo, bajo este criterio, se separa y excluye a Pablo Guevara (Lima, 1930-2006) como un representante de la generación del 50 cuando su obra evidencia mayor relación con la generación del 60; solo por citar dos ejemplos emblemáticos, efectos de la cuadriculación por décadas.

Los principios que constituyen las generaciones, planteados tanto por Pinder como por Petersen, quedan también desvirtuados. En primer lugar, porque no se pueden sectorizar las generaciones en reuniones gremiales de poetas, por ejemplo, sino en una idea que abarque diferentes personalidades de diversos ámbitos culturales, políticos y sociales que reúnan convergencias en educación y que se aprecien marcados por eventos que los cohesionen y orienten hacia una mirada común de un determinado momento, bajo la conducción de un guía. En este sentido, ¿quién fue el guía para la generación del 60 como lo fue, en opinión de muchos, Salazar Bondy o, para otros, Jorge Puccinelli para la generación del 50? ¿Tal vez haya sido Sologuren o solo para los poetas de «La Rama Florida»? ¿Y quién asume el rol durante la del 70 y la del 80, de por sí iconoclastas? ¿La generación del 90, tan individualista, tiene algún guía? ¿Luis Jaime Cisneros será el guía de referencia común de tantos coetáneos de todas estas generaciones? ¿Todos los poetas han recibido 
https://doi.org/10.46744/bapl.201901.001

similar educación o qué aspectos los cohesionan política o socialmente? ¿Si segmentamos a los poetas, tendrán vinculación directa con sus demás coetáneos, sean artistas o no? Todos estos puntos fundamentales en la teoría de las generaciones hacen agua en la periodización actual de nuestra lírica de la segunda mitad del siglo xx.

Quizá la voz de Carlos García Bedoya sea la más escéptica y lúcida de las que denuncian el mal uso del concepto. Para el historiador de la literatura peruana, el término «generación» no representa una unidad orgánica, sino aparente y que, además, debe ser considerada como una parte entre los muchos factores que constituyen la personalidad literaria de un autor. Por ello, la categoría «generación» cumple un rol más descriptivo, que se puede aplicar con utilidad cuando no se dispone de la suficiente distancia estética para observar a un grupo de escritores recientes, "cuando la proximidad entre el observador y el objeto de estudio impide percibir con claridad las varias orientaciones que coexisten en ese todo empírico que llamamos generación» (García Bedoya, 2004: 24). Es evidente la instrumentalización del concepto que para el catedrático peruano «no resulta pertinente para el estudio sistemático de la evolución literaria». El problema de la asignación de «generación» a un vacío de reflexión conceptual de teoría literaria no solo se presenta en nuestro país, sino también en otros lugares de Latinoamérica; sobre todo, en Colombia y México, donde los nombres llegan al absurdo de ser referentes subjetivos en lugar de la identificación por décadas. ${ }^{1}$ A pesar de todo esto, cabe considerar que el término «generación» resulta útil cuando se aplica a un sector de autores porque no se puede apreciar la naturaleza del momento de producción determinado con la suficiente distancia temporal. Es decir, el uso del concepto en sí mismo es válido y hasta necesario, pero su abuso, como toda medida en exceso, desvirtúa su carácter. A veinte años de distancia del inicio del siglo xxi y a setenta años de la generación del 50 que inicia esta serie, es fundamental encontrar una alternativa a esta aplicación.

1 Véanse los comentarios de Patricia Trujillo (2003) para algunos nombres como La generación posnadaísta o La generación sin nombre en Colombia, y los de Samuel Gordon (2004) para denominaciones como La generación del medio siglo en México. 
En segundo lugar, una mirada panorámica a la crítica e historia de nuestra lírica nos ofrece una serie mezclada de conceptos con los que se ha tratado de asociar o de identificar manifestaciones que marcan el derrotero de la poesía peruana del siglo xx. Por ejemplo, se destaca el modernismo (corriente literaria, ¿acaso movimiento literario?), el posmodernismo (irealmente una corriente o simplemente una tendencia en la que evoluciona la poesía modernista sin abandonar las bases ordenadas de su(s) estilo(s) constitutivo(s)?), la vanguardia (iescuelas como en Europa o, a excepción del surrealismo en poetas como Moro o Westphalen, una fusión de elementos vanguardistas unida a una determinada actitud?) y, quizá, podamos incluir peregrinos nombres como el «cholismo» para reconocer en todos ellos marcas nominales de las diferentes expresiones líricas de la primera mitad del siglo xx. En cuanto a la segunda mitad, esta se encuentra determinada — como ya se sostuvo- por el concepto de «generación». De tal modo, el caos en la forma en que se determina el carácter de la poesía peruana contemporánea es evidente. Los conceptos se aplican sin orden, saltando entre corrientes, movimientos, escuelas, pseudoperiodos y generaciones que no describen el desarrollo del fenómeno, sino que lo confunden arbitrariamente en esta mixtura de términos.

Adviértase el problema de este punto: esta imagen incompleta se difunde y reproduce desde los libros escolares hasta las enciclopedias e historias de la literatura peruana que constituyen ejes de divulgación fundamentales para la mayoría de los peruanos que acude a ellos, justamente, porque no orientará su vida a la reflexión literaria de forma profesional. De esta manera, una imagen borrosa de su acervo cultural poético queda impresa en la mayoría de los compatriotas. Al parecer, la tendencia a interpretar movimientos y colectivos de época de la primera mitad del siglo xx pierde sentido cuando se enfoca en la segunda mitad de dicho siglo. Evidentemente, la respuesta a esta natural disposición no se encuentra en el salvavidas del concepto «generación».

En tercer lugar, surge el vacío en la reflexión teórica e histórica, exclusiva y directa, sobre la evolución espontánea de nuestra poesía. Esto determina la paupérrima situación en la que nos encontramos. Al inicio de mi investigación, mientras recopilaba información y determinaba el corpus 
https://doi.org/10.46744/bapl.201901.001

de reflexión sobre la poesía peruana y su evolución, pude constatar que no existía un libro que la trabajara exhaustivamente como un fenómeno que se desarrolla de forma espontánea, sino que los críticos, historiadores e investigadores se concentran en movimientos o conceptos como los que determinan el caos en el régimen de términos que he descrito. Así, queda parcelada y anquilosada la apreciación de un fenómeno, más bien, dinámico. Hay publicaciones —incluso actuales — sobre la poesía modernista, sobre la poesía vanguardista y sobre las generaciones de poetas. Sin embargo, el último libro que se propuso considerar exclusivamente la producción poética como objeto de reflexión (con el ojo en su dinamismo) fue el de Luis Monguió, La poesía postmodernista peruana (1954). En esta obra, el crítico español explica la evolución desde los ecos del romanticismo hasta la poesía que se compone en su momento después del modernismo, y considera estas últimas tendencias bajo la denominación de "poesía postmodernista» (a falta de un nombre que englobe las circunstancias de su tiempo). Este endeble término «bisagra» entre dos periodos claros de nuestra lírica (el modernismo y la vanguardia) pudo haber sido útil en su momento, pero se repitió irreflexivamente, con lo que se generó una confusión de valores o jerarquías conceptuales que desencadenó la idea de un periodo postmodernista que resulta precario porque su expresión poética no se aleja lo suficiente de los principios del modernismo ni dura lo necesario para considerar un cambio determinante en la praxis de nuestra poesía de entonces. Si bien este no es el sentido que le otorgó Luis Monguió, es el sentido que ha marcado la huella de historiadores como Augusto Tamayo Vargas que asentaron esta interpretación del postmodernismo que — como ya se ha manifestado — se difunde desde la escuela debido a la popularidad que alcanzan sus reflexiones. Tomando en cuenta a Abraham Valdelomar (solamente en su producción poética y no en su narrativa), se puede evaluar qué tanto se distancia de los preceptos y del estilo musical —ordenado y, muchas veces, alturado- del modernismo. Considerando, además, que el modernismo de José Martí incluye lo cotidiano y sencillo, parece un despropósito encasillar al autor de «Tristitia» en los márgenes de un periodo distinto, postmodernista, con semejante jerarquía que el modernista, simplemente porque, en sus poemas, se encuentran algunos aspectos cotidianos, sencillos, realistas que se alejan del exotismo, simbolismo u onirismo de Darío. 
La reflexión directa sobre la evolución del fenómeno poético se encuentra, en su mayoría, en las antologías y en las historias de la literatura peruana. Por ello, se trata de una aproximación parcial y, en algunos casos, hasta tangencial porque, en las historias, por ejemplo, prima más la articulación del fenómeno a partir del autor o a partir de la perspectiva contextual de sus obras en conjunto — sean o no poemas - que la posibilidad de señalar, efectivamente, el rol que cumple su producción poética en una evolución netamente lírica. Por ejemplo, a pesar de explicarse que Manuel González Prada fue un poeta modernista, casi todas las historias, que aspiran a englobar toda su producción - y no solo la lírica - repiten su encasillamiento en el realismo. Esto es correcto, aunque impreciso. Si hubiese una historia exclusiva que revise la poesía peruana, muchos matices y aspectos ambiguos quedarían fijados o esclarecidos, sobre todo, si se quiere (re)valorar este campo tan importante de la literatura. De igual manera, debido a su propia naturaleza, las antologías registran y configuran también a los poetas que representan el canon y, por ello, resulta ocioso exigirles a estos libros que señalen con precisión su perspectiva sobre el dinamismo lírico en el que se encuentran imbuidos los autores seleccionados. Cuando una antología se orienta a un momento de la poesía, también comete el error de circunscribirse a los inamovibles conceptos que cuestionamos. Así, se ofrecen antologías sobre la base de generaciones (Los nuevos, de Leonidas Cevallos; Estos 13, de José Miguel Oviedo; La última cena, para la generación del 80 o Los relojes se han roto, de Bernales y Villacorta, para la del 90) que, justamente, las promueven; antologías sobre la vanguardia (la famosa de Eielson, Sologuren y Salazar Bondy, de 1946; la de Lauer, de 2001, o la de Luis Chueca, PUCP, 2009) o sobre el modernismo (Las voces múltiples, de 1916) o antologías desde una perspectiva general que no especifican la ubicación de los poetas reunidos (Poesía peruana 50 poetas del siglo xx, 2001, de Carlos Garayar) o que los determina, una vez más, bajo los conceptos cuestionados (Poesía peruana contemporánea. Antología crítica, 2002-2003, de Toro Montalvo). El espíritu del libro de Luis Monguió no se ha vuelto a repetir para interpretar la segunda mitad del siglo xx de nuestra lírica $y$, quizá, por ello, naufragamos en un mar de generaciones.

La poca reflexión sobre la evolución de la poesía contemporánea silencia el debate y promueve la repetición autómata de un viejo canon 
que reposa sobre los soportes de otro horizonte de expectativas, caduco para una mirada desde el siglo xxi. La difusión de revistas, libros escolares, diccionarios y demás estudios mantiene la inamovilidad histórica de este fenómeno variable poco asible que se figura como la estela de nuestra lírica peruana.

En cuarto lugar, puedo afirmar, sin duda, que la propuesta de mi tesis doctoral no descubre la pólvora; sino que, sencillamente, se sirve de un concepto que, si bien posee larga data, aún resulta funcional y permite recuperar un orden en la percepción del desarrollo de la poesía peruana del siglo xx. Sobre la base del concepto "periodo", interpreto esta evolución de manera exclusiva del género lírico. ${ }^{2} \mathrm{Al}$ observar el siglo xx, en realidad, debido a la misma manifestación móvil en los estilos de la producción poética, se incluyen los últimos años del siglo XIX, así como estos casi veinte años de lo que va del siglo xxi, porque las tendencias se imbrican más allá de los cambios en el calendario. En este sentido, mi propuesta consiste en establecer cuatro periodos en la poesía peruana contemporánea:

\begin{tabular}{|l|l|l|}
\hline Primer periodo & 3 etapas & Asimilado al modernismo \\
\hline Segundo periodo & 3 etapas & $\begin{array}{l}\text { Incluye la vanguardia, los años } \\
\begin{array}{l}\text { de y } 40 \text { así como la generación } \\
\text { del } 50\end{array}\end{array}$ \\
\hline Tercer periodo & Sin etapas & $\begin{array}{l}\text { Sustituye las llamadas genera- } \\
\text { ción del } 60,70 \text { y } 80\end{array}$ \\
\hline Cuarto periodo & Sin etapas & $\begin{array}{l}\text { Comprende desde la denominada } \\
\text { generación del } 90 \text { hasta nuestros } \\
\text { días }\end{array}$ \\
\hline
\end{tabular}

2 Para determinar las características del concepto «periodo» he revisado las teorías literarias clásicas de Warren y Wellek (1942), Aguiar e Silva (1979), así como las perspectivas teóricas más modernas como la de Claudio Guillén (Entre lo uno y lo diverso, 1985), además de la reflexión de autores peruanos como Carlos García Bedoya Maguiña (Para una periodización de la literatura peruana, 2004). 
Para establecer estas marcas temporales, he tomado los conceptos emergente, dominante y residual de la teoría de Raymond Williams (2001) con la que se explican los desarrollos culturales de una sociedad. Ya que cuando se aborda especialmente la poesía, lograr precisiones absolutas resulta casi imposible — cuando no improductivo-, los periodos se establecen sobre márgenes relativos que se determinan en un momento concreto y considerando la heterogeneidad de las manifestaciones, a partir de la significación de la publicación de un poemario fundamental - $\mathrm{O}$, en el caso, del segundo periodo de un hecho como el cierre de dos publicaciones deliberantes - para establecer los cambios en las convenciones artísticas, literarias o poéticas. A estas publicaciones, las asumo como hitos que sirven más como referentes que como modelos, paradigmas o representaciones jerárquicas. De esta manera, los periodos se pueden reconocer por un carácter emergente a partir de los siguientes hitos:

\begin{tabular}{|l|l|l|}
\hline Primer periodo & 3 etapas & $\begin{array}{l}\text { Primera etapa: Minúsculas } \\
\text { (1901) Manuel González Prada } \\
\text { Segunda etapa: Alma América } \\
\text { (1906) José Santos Chocano, } \\
\text { hasta Simbólicas (1911) José } \\
\text { María Eguren } \\
\text { Tercera etapa: Los heraldos negros } \\
\text { (1918) César Vallejo }\end{array}$ \\
\hline Segundo periodo & 3 etapas & $\begin{array}{l}\text { Incluye la vanguardia, los años } \\
\text { 30 y 40 así como la generación } \\
\text { del 50 } \\
\text { Primera etapa: Trilce (1922) Cé- } \\
\text { sar Vallejo } \\
\text { Segunda etapa: Amauta y el Bo- } \\
\text { letín Titikaka (1930) } \\
\text { Tercera etapa: Reinos (1945) } \\
\text { Jorge Eduardo Eielson }\end{array}$ \\
\hline
\end{tabular}


https://doi.org/10.46744/bapl.201901.001

\begin{tabular}{|l|l|l|}
\hline Tercer periodo & Sin etapas & $\begin{array}{l}\text { Sustituye las llamadas genera- } \\
\text { ción del 60, 70 y 80 } \\
\text { Comentarios reales (1964) Anto- } \\
\text { nio Cisneros }\end{array}$ \\
\hline Cuarto periodo & Sin etapas & $\begin{array}{l}\text { Comprende desde la llamada } \\
\text { generación del 90 hasta nues- } \\
\text { tros días } \\
\text { Symbol (1991) Roger Santiváñez }\end{array}$ \\
\hline
\end{tabular}

Desde nuestro actual horizonte de expectativas, revisé la evolución y determiné las características no excluyentes - ni tampoco exclusivas, sino preponderantes - de cada uno de los cuatro periodos con sus exponentes ejemplares o hegemónicos (que no implican un juicio de valor o una elección orientada al gusto subjetivo). La elección de estos últimos se debe a su inserción en el canon a través de la mención que se hace de ellos en un corpus extenso de historias y antologías que componen parte de la bibliografía de mi investigación. Así, el canon se configura sobre la fórmula de una reiteración, quizá, más que debido a una evaluación o cuestionamiento constante (o, al menos, eventual). Este método se encuentra en consonancia con el espíritu de mi tesis que se concentra en revisar (y cuestionar) la periodización que ya existe sobre nuestra poesía del siglo xx.

El primer periodo coincide con lo que concebimos hasta ahora como modernismo. Por lo tanto, mi propuesta no socaba las convenciones culturales que se arraigan sobre las bases del movimiento. Este periodo se encuentra caracterizado por la producción ecléctica, el abanderamiento en la subjetividad de lo moderno, la innovación dentro de los parámetros clásicos de la rítmica y métrica, la influencia de José Martí, Rubén Darío y de los poetas simbolistas franceses. Posee tres etapas — en lo que coincido con el crítico Ricardo González Vigil (2004)—, de las cuales la tercera se asumiría como aquello que consideramos bajo el término posmodernismo: etapa residual de este periodo y no un nuevo periodo autónomo previo 
a la vanguardia con una jerarquía mayor. Los autores canónicos que por excelencia representan nuestro modernismo son: Manuel González Prada, José Santos Chocano, José María Eguren, Abraham Valdelomar y el primer César Vallejo. En segunda instancia, las historias y antologías de este primer periodo registran en su mayoría los siguientes principales autores canónicos: José Eufemio Lora y Lora, José Gálvez Barrenechea, Ventura García Calderón, Domingo Martínez Luján, Enrique A. Carrillo, Leonidas Yerovi, Luis Fernán Cisneros, Alberto Ureta, Juan Parra del Riego, Enrique Bustamante y Ballivián, Percy Gibson, César A. Rodríguez, Alcides Spelucín. ${ }^{3}$

El segundo periodo abarca aquello que entendemos por vanguardia e incluye la, relativamente innominada, expresión poética que se produjo durante los años treinta y cuarenta, así como la denominada generación del 50. También se aprecia subdividido en tres etapas: de 1922 a 1930, de 1930 a 1945, y de 1945 a 1964; fechas que, evidentemente, no marcan el fin absoluto de la progresión de este periodo, sino que evidencian sus intersecciones con aquel que lo precede y con el que le sigue. Se trata, por lo tanto, de un momento extenso que une espíritus de renovación artística que se extiende desde la vanguardia de los años veinte hasta las producciones de los poetas de la generación del 50. Dicha unidad, relación, convergencia o confluencia de la vanguardia y la generación del 50 se sustenta en las investigaciones de varios autores como Luis Rebaza Soraluz (2017), por citar un caso reciente. Por lo tanto, este segundo periodo se caracteriza esencialmente por reasumir los conceptos de corrientes y movimientos como la vanguardia, el cholismo, el nativismo, la generación del 50, y, por lo mismo, se encuentra determinado por un carácter transculturador. La innovación se inicia con un rompimiento de los marcos convencionales de rítmica y métrica en la lírica y mantiene una dirección en busca de la originalidad de la tradición nacional entre los cauces de lo social y lo esencialista, de lo occidental y lo andino. En cuanto a los representantes, debido a la extensión

3 Debido al dinamismo de la evolución de la poesía, autores como Parra del Riego y Bustamante y Ballivián, por ejemplo, se asumirán también como vanguardistas debido al desarrollo de su trabajo poético. 


\section{propuesta, hay divergencias que se deben considerar según la fuente que los determine. ${ }^{4}$}

\section{El tercer periodo fusiona lo que, con ligereza, denominamos} generación del 60, del 70 y del 80. Estas tres generaciones asimilan un proceso histórico fundamental para nuestro país que se refleja en

$4 *$ Los principales representantes de este segundo periodo según las historias son los siguientes. Primera etapa: César Vallejo, Alberto Hidalgo, Carlos Oquendo de Amat, Alejandro Peralta. Segunda etapa: Xavier Abril, Emilio Adolfo Westphalen, César Moro, Martín Adán, José María Arguedas, Enrique Peña Barrenechea, Mario Florián, Manuel Moreno Jimeno. Tercera etapa: Jorge Eduardo Eielson, Javier Sologuren, Sebastián Salazar Bondy, Gustavo Valcárcel, Blanca Varela, Alejandro Romualdo, Wáshington Delgado, Carlos Germán Belli, Juan Gonzalo Rose, Francisco Bendezú, Leopoldo Chariarse, Pablo Guevara, Manuel Scorza. Con menor recurrencia: (1. ${ }^{a}$ etapa) Magda Portal, Emilio Armaza, Reynaldo Bolaños (Serafín Delmar), Guillermo Mercado, Gamaliel Churata, Juan Luis Velásquez, Federico Bolaños, Luis A. Rodríguez (Luis de Rodrigo), Juan José Lora, Alberto Guillén, Emilio Vásquez, Mario Chabes; (2. ${ }^{a}$ etapa) Juan Parra del Riego, José Alvarado Sánchez, Luis Nieto, Julio Garrido Malaver, Nicanor de la Fuente (Nixa), Juan Ríos, Luis Valle Goicochea, Alberto Cuentas Zavala, Augusto Tamayo Vargas, José Alfredo Hernández, Luis Fabio Xammar, José Varallanos, Adalberto Varallanos, Óscar Bolaños (Julián Petrovick); (3. ${ }^{a}$ etapa) Leoncio Bueno, José Ruiz Rosas, Pedro Cateriano, Cecilia Bustamante, Demetrio Quiroz Malca, Yolanda Westphalen, Sarina Helfgott, Augusto Elmore, Alberto Escobar, Marco Antonio Corcuera, Eleodoro Vargas Vicuña.

* Los principales representantes de este segundo periodo según las antologías generales son los siguientes. Primera etapa: César Vallejo, Carlos Oquendo de Amat, Alberto Hidalgo, Alejandro Peralta, Luis Valle Goicochea. Segunda etapa: Xavier Abril, Emilio Adolfo Westphalen, César Moro, Martín Adán, Enrique Peña Barrenechea, Mario Florián. Tercera etapa: Jorge Eduardo Eielson, Javier Sologuren, Sebastián Salazar Bondy, Blanca Varela, Wáshington Delgado, Juan Gonzalo Rose, Pablo Guevara. Con menor recurrencia: (1. ${ }^{a}$ etapa) Gamaliel Churata, Guillermo Mercado; (2. ${ }^{a}$ etapa) Manuel Moreno Jimeno, Ricardo Peña, Vicente Azar, Juan Ríos, Luis Nieto, Julio Garrido Malaver, José María Arguedas, Augusto Tamayo Vargas; (3. ${ }^{a}$ etapa) Gustavo Valcárcel, Alejandro Romualdo, Carlos Germán Belli, Francisco Bendezú, Leoncio Bueno, Leopoldo Chariarse, Cecilia Bustamante, Manuel Scorza, Yolanda de Westphalen.

* Los principales representantes de este segundo periodo según las antologías especializadas en cada etapa son los siguientes. Primera etapa: César Vallejo, Carlos Oquendo de Amat. Segunda etapa: Martín Adán, Xavier Abril, Enrique Peña Barrenechea. Tercera etapa: Francisco Bendezú, Wáshington Delgado, Juan Gonzalo Rose, Carlos Germán Belli. Con menor recurrencia: (1. ${ }^{\text {a }}$ etapa) Alejandro Peralta, Magda Portal, Alberto Hidalgo, Juan Luis Velázquez, Emilio Armaza, Federico Bolaños, Alberto Guillén, Enrique Bustamante y Ballivián, Juan José Lora, Serafín Delmar, Mario Chabes, César Atahualpa Rodríguez; (2. ${ }^{a}$ etapa) Emilio Adolfo Westphalen, Julián Petrovick, José Varallanos, Juan Parra del Riego, César Miró; (3. a etapa) Jorge Eduardo Eielson, Javier Sologuren, Sebastián Salazar Bondy, Gustavo Valcárcel, Demetrio Quiroz-Malca, Blanca Varela, Alejandro Romualdo, Leopoldo Chariarse, Pablo Guevara, Manuel Scorza. 
los textos poéticos y que se inscribe entre las guerrillas y el final de las acciones de Sendero Luminoso, por lo que abarca la década de 1990. Se encuentra caracterizado sobre la base de la poesía conversacional y el coloquialismo, por orientarse hacia la perspectiva de lo urbano, la descomposición social y la violencia. Por ello, el componente social y la objetivación y narratividad de la lírica en este periodo es evidente. Asimismo, se aprecia una influencia de la poesía anglosajona, la emergencia de la lírica femenina y una tendencia gregaria en la manifestación de los poetas canónicos. Algunos autores continúan la línea experimental y, alentados por la libertad "poundiana» del concepto de poesía, integran discursos no tan —clásicamentelíricos con sus tendencias narrativas. Por ello, se aprecian fórmulas, proposiciones matemáticas y otros signos. Esto se verá de manera más radical, sin embargo, en el siguiente periodo. Asimismo, se observan las poéticas de autores como José Watanabe, Antonio Cillóniz, Eduardo Chirinos, Alfonso Cisneros Cox, Carlos López Degregori, Renato Sandoval, Odi Gonzales, Rossella Di Paolo, entre otros, que se alejan de la hegemonía del periodo y amplían la heterogeneidad del mismo con sus particulares estilos. Cabe incluir en la lista de representantes, autores como Arturo Corcuera, Pablo Guevara, José María Arguedas y Jorge Wiesse, quienes por cuestiones de fechas (de nacimiento o de publicación) se encuentran normalmente alejados de este periodo que naturalmente los convoca. ${ }^{5}$

$5 \quad *$ Las historias de la literatura consagran a los siguientes poetas principales de este tercer periodo como parte del canon por su mención reiterativa: Antonio Cisneros, Rodolfo Hinostroza, Luis Hernández, Javier Heraud, César Calvo, Livio Gómez, Arturo Corcuera, Marco Martos, Juan Ojeda, Hildebrando Pérez, Antonio Cillóniz, José Watanabe, Abelardo Sánchez León, Enrique Verástegui, Jorge Pimentel, Manuel Morales, Carlos Zúñiga Segura, Juan Ramírez Ruiz, Armando Rojas, Jorge Nájar, César Toro Montalvo, Carmen Ollé, Tulio Mora, José Rosas Ribeyro, María Emilia Cornejo, Nicolás Yerovi, José Luis Ayala, Segundo Cancino, Beethoven Medina, Carlos López Degregori, Alfonso Cisneros Cox, Jorge Eslava, Eduardo Urdanivia Bertarelli, Giovanna Pollarolo, Oswaldo Chanove, Alonso Ruiz Rosas, Róger Santiváñez, Eduardo Chirinos, José Antonio Mazzotti, Rocío Silva Santisteban y José Morales Saravia.

* Las antologías generales, reiteran a los representantes principales de este periodo como: Marco Martos, César Calvo, Antonio Cisneros, Rodolfo Hinostroza, Livio Gómez, Juan Ojeda, Winston Orrillo, Javier Heraud, Arturo Corcuera, Luis Hernández, Ricardo Silva Santisteban, Mirko Lauer, Armando Rojas, José Watanabe, Tulio Mora, Enrique Verástegui, César Toro Montalvo, Jorge Pimentel, Manuel Morales, Danilo Sánchez Lihón, 
El cuarto periodo expresa un cambio tanto en la forma de producir como en la de consumir poesía. En relación con esto, se asienta la percepción de aquello que se ha difundido como posmodernidad (Lyotard, 1987) y sus variantes como la hipermodernidad (Lipovetsky, 2014). Este periodo se caracteriza, esencialmente, por la diversidad y lo difuso que se encuentran en una poética de integración o convergencia. La simultaneidad y la fragmentación modernas se radicalizan para alcanzar el inmediatismo, es decir, se trata de refundar los principios de la modernidad. La relativización de cualquier concepto hace eco de «la caída de los grandes relatos» en pro de la individualidad en las válidas pluralidades. Asimismo, los paradigmas del sistema se han transformado, con tanta evidencia, que la crítica se contrae en mutismo o en un juicio sin herramientas de valoración actualizadas. Finalmente, se nota una ausencia de movimientos o escuelas que se aprecian en la adecuación del concepto de «modernidad» en «posmodernidad» (individualista, relativista), es decir, en la diversidad, heterogeneidad y en lo difuso de la producción poética actual, a pesar de los colectivos y agrupaciones efímeras.

A pesar de la poca solidez en las publicaciones críticas sobre los representantes de este periodo (inclúyase la falta de antologías y de

Juan Ramírez Ruiz, Jorge Nájar, Abelardo Sánchez León, José Rosas Ribeyro, Eduardo Chirinos, Róger Santiváñez, Mario Montalbetti, Carlos Guevara, Rossella Di Paolo, Édgar O'Hara, Eduardo Urdanivia Bertarelli, Luis Alberto Castillo, Carlos López Degregori, Fernando Castro Ramírez, Giovanna Pollarolo, José Pancorvo, Enrique Sánchez Hernani, Jorge Eslava Calvo, José Morales Saravia, Oswaldo Chanove, Luis Rebaza, José Antonio Mazzotti, Carlos Reyes Ramírez, Juan de la Fuente y Rocío Silva Santisteban.

* Las antologías que consignan autores de la segunda mitad del siglo XX consideran a los siguientes representantes principales del tercer periodo: Domingo de Ramos, Jorge Pimentel, Cesáreo Martínez, Juan Ramírez Ruiz, Abelardo Sánchez León, Carmen Ollé, Enrique Verástegui, Róger Santiváñez, Eduardo Chirinos, Rossella Di Paolo, Rocío Silva Santisteban y Jorge Frisancho.

* Las antologías por generaciones reconocen a los siguientes autores principales dada su reiteración: Antonio Cisneros, Marco Martos, Julio Ortega, Carlos Henderson, César Calvo, Winston Orrillo, Javier Heraud, Mercedes Ibáñez Rosazza, Luis Enrique Tord, Raúl Bueno, Rodolfo Hinostroza, Mirko Lauer (poetas del sesenta); Juan Ramírez Ruiz, Feliciano Mejía, Tulio Mora, José Rosas Ribeyro, José Cerna, Enrique Verástegui, Manuel Morales (poetas del setenta); Eduardo Chirinos, José Antonio Mazzotti, Magdalena Chocano, Mariela Dreyfus, Oswaldo Chanove, Rocío Silva Santisteban, Róger Santiváñez, Rossella Di Paolo, Domingo de Ramos y Jorge Frisancho (poetas del ochenta). 
expansión en las historias), los nombres reiterados de los principales poetas son José Carlos Yrigoyen, Miguel Ildefonso, Rafael Espinosa, Xavier Echarri, Roxana Crisólogo, Carlos Oliva, Victoria Guerrero, Montserrat Álvarez, Lorenzo Helguero, Josemári Recalde, Martín Rodríguez Gaona, Rubén Quiroz, José Pancorvo, Andrea Cabel, Giancarlo Huapaya, Javier Gálvez y Alberto Valdivia Baselli. Y con menor presencia, Víctor Coral, Alessandra Tenorio, Salomón Valderrama, Diego Lazarte, Miguel Ángel Malpartida, Víctor Ruiz, Gonzalo Portals Zubiate, José Beltrán Peña, Lizardo Cruzado, Selenco Vega Jácome, Josefina Barrón, José Gabriel Cabrera, Carolina Fernández, Andrés Piñeiro, Edgar Saavedra, Alejandra del Valle, Marita Troiano, Manuel Fernández, Willy Gómez Migliaro, Paul Guillén, Orlando Granda, Jorge Trujillo, Elio Vélez, Ricardo Ayllón, Antonio Sarmiento, Diego Otero, Alonso Rabí, Roberto Zariquiey, Rafael García Godos Salazar.

El problema de fondo — quizá irresoluble — de esta periodización se encuentra en asignar un nombre a cada periodo que cale tan hondo como los conceptos cuestionados y que, a la vez, represente el espíritu de la producción de cada época. Por eso, se podría acceder a nombrar el primer periodo como el periodo del modernismo; al segundo, como el periodo de vanguardismo (que no se acepte ni confunda con el concepto de vanguardia); al tercero, como el periodo de la heterodoxia (acorde con el planteamiento de Alberto Escobar, 1973, sobre los poetas que cuestionan la tradición), y al cuarto, como el periodo de la posmodernidad (que no debe confundirse con el posmodernismo de la primera mitad del siglo xx, ya cuestionado en su carácter de pseudo periodo). A estas nominaciones hay que identificarlas más con caracterizadores nombres conceptuales que con otra mezcolanza de movimientos, corrientes o términos dispares.

$\mathrm{Al}$ proponer una tesis como esta surgirán, por supuesto, algunas objeciones razonables que se estipulen desde una rígida mirada contra lo dinámico. Como adelanto a algunas de ellas, cabe sostener que el mismo trabajo mide sus limitaciones. En primer lugar, como muchos estudios, este se encuentra restringido a la poesía editada, escrita, 
https://doi.org/10.46744/bapl.201901.001

expresada en castellano y que corresponde, en su mayoría, al sistema culto (Cornejo Polar, 2013). Esto es así, no solo por las limitaciones del investigador, sino también por tratarse de un reflejo de aquello que como se indica en el título de la tesis- corresponde con la revisión del canon. De esta manera, esta tesis denuncia también los problemas que surgen de la poca investigación y difusión de autores no canónicos, de producciones en otros idiomas, de naturaleza oral. En segundo lugar, se aprecia la noción de poesía que se maneja y la derivación en el nombre de lírica que ha llegado a popularizarse hasta hoy como poesía en general. En tercer lugar, se trata de un cuestionamiento a la ideología que se manifiesta como sustrato de todo canon y de un estímulo para reivindicar el carácter dinámico que posee la lírica si se revisa exclusivamente en su «exacta dimensión». Por supuesto, se objetará el sentido de esta periodización si se puede graficar el tema desde la percepción de cada autor, de su nacionalidad, del género o de algún otro factor; pero insisto en la ventaja de realizarlo desde el concepto de "periodo", que no debe entenderse como imposición, reducción u homogenización a unas pocas características de algo que pretende ser, en suma, heterogéneo y único como cada poema. Desde el periodo, se percibe directamente el carácter dinámico del fenómeno poético a analizar y se estudia su desarrollo teniendo a la poesía misma como protagonista. En cuarto lugar, algunas irregularidades que arrastramos hasta hoy podrían, finalmente, desterrarse de nuestro discurso, comenzando por la automática reproducción del término «generación», la interpretación de un periodo llamado «posmodernismo» antagónico a los patrones del modernismo en la lírica, y la división estéril entre poetas puros y sociales o comprometidos que se radicaliza durante la generación del 50.

Finalmente, el espíritu de este trabajo consiste en abrir el debate sobre el canon. Asimismo, pretende hacer hincapié en la importancia de mantener la reflexión sobre la lírica y en postular un esquema de cuatro periodos que puedan aplicarse y explicarse desde los estudios escolares de secundaria para difundir un fenómeno ordenado en conceptualizaciones. Este esquema refleja una coherente aproximación y se dirige a todo público que desee entender la 


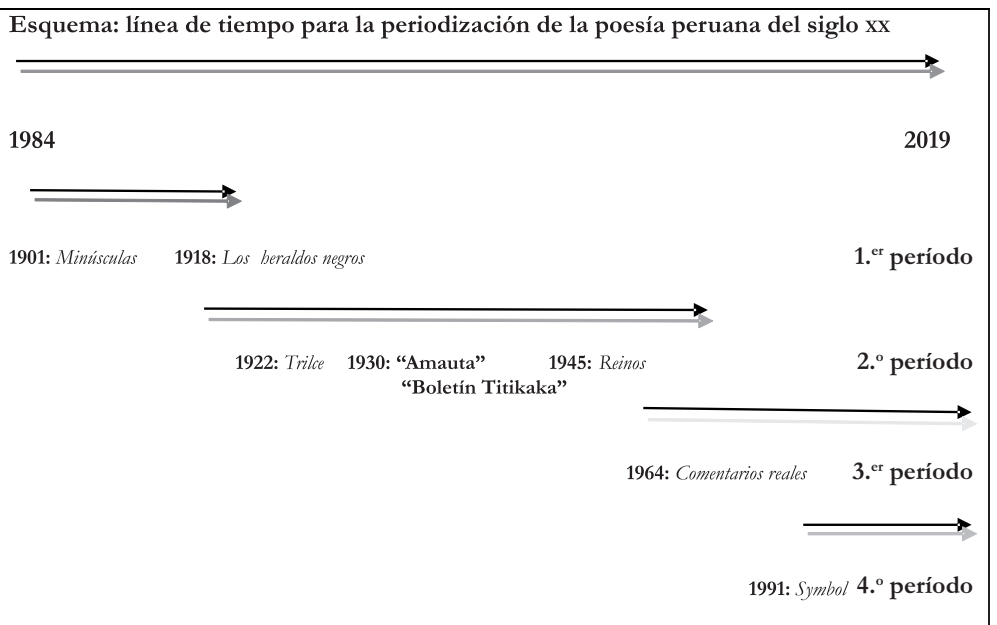


evolución de nuestra poesía peruana contemporánea, sin lugar a dudas, la mejor de nuestra historia.

\section{BIBLIOGRAFÍA}

AGUIAR E SILVA, V. (1982). Teoría de la literatura. Madrid: Gredos.

BERNALES, E., VILLACORTA, C. (Ed). (2005). Los relojes se han roto. Antología de poesía peruana de los noventa. México: Arlequín.

CEVALLOS, L. (Ed). (1967). Los nuevos. Lima: Universitaria.

CHUECA, L. (Ed). (2009). Poesía vanguardista peruana. Lima: PUCP.

CORNEJO POLAR, A. (2013). «La literatura peruana: totalidad contradictoria». En Crítica de la razón heterogénea. Textos esenciales I (pp. 51-73). Lima: Asamblea Nacional de Rectores.

EIELSON, J., SOLOGUREN, J., SALAZAR BONDY, S. (Ed). (2013). La poesía contemporánea del Perú. Lima: Biblioteca Abraham Valdelomar.

ESCOBAR, A. (Ed). (1973). Antología de la poesía peruana. Lima: Peisa.

GARAYAR, C. (Ed). (2001). Poesía peruana 50 poetas del siglo XX. Lima: Peisa/EL Comercio.

GARCÍA-BEDOYA MAGUIÑA, C. (2004). Para una periodización de la literatura peruana. Lima: UNMSM.

GONZÁLEZ VIGIL, R. (2004). Literatura. En Enciclopedia temática del Perú. Tomo XIV. Lima: El Comercio. 
https://doi.org/10.46744/bapl.201901.001

GORDON, S. (2004). Breves atisbos metodológicos para el examen de la poesía mexicana al fin de siglo. Recuperado de http:// www.google.com.pe/url? sa $=t \& r c t=j \& q=\& \operatorname{esrc}=s \&$ source $=$ web\&cd $=1 \& v e d=0 C B s Q F j A A \& u r l=h t t p$ $\% 3 \mathrm{~A} \% 2 \mathrm{~F} \% 2 \mathrm{Ffiles}$.tallerdepoesiacolonense.webnode. es\%2F200000024-730c574fe7\%2FGordon\%2520Ati bos\%2520Metodol\%25C3\%25B3 gicos....pdf\&ei $=\mathrm{gR}$ ObVcDgA4yyggTw36roCA\&usg $=$ AFQjCNGoBa_m v5GtQoD5OVRjdOEQk17sA

GUILLÉN, C. (2018). Entre lo uno y lo diverso. Barcelona: Austral.

LAUER, M. (Ed). (2001). Antología de la poesía vanguardista peruana Lima: El Virrey.

LIPOVETSKY, G., Charles S. (2014). Los tiempos hipermodernos. Barcelona: Anagrama.

LYOTARD, J. (1987). La condición postmoderna: informe sobre el saber. Madrid: Cátedra.

ORTEGA Y GASSET, J. (2012). En torno a Galileo. En Ortega y Gasset Biblioteca de grandes pensadores Madrid: Gredos.

OVIEDO, J. (Ed). (1973). Estos 13. Lima: Mosca Azul.

PETERSEN, J. (1946). Las generaciones literarias. En Ermatinger, E. (Ed). Filosofía de la ciencia literaria. México: FCE.

PINDER W. (1946). El problema de las generaciones en la bistoria del arte de Europa. Buenos Aires: Losada.

REBAZA SORALUZ, L. (2017). De ultramodernidades y sus contemporáneos Lima: FCE. 
https://doi.org/10.46744/bapl.201901.001

TORO MONTALVO, C. (Ed) (2002-2003). Poesía peruana contemporánea. Antología crítica. Lima: Universidad Inca Garcilaso de la Vega.

TRUjILlO, P. (2003). Periodos y generaciones en la historia de la poesía colombiana del siglo $X X$. Recuperado de

http://www.google.com.pe/url? sa $=t \& r c t=j \& q=\&$ esrc $=$ s\&source $=w e b \& c d=2 \&$ ved $=0 C C E Q F j A B$ \&url $=\mathrm{http} \% 3 \mathrm{~A} \% 2 \mathrm{~F} \% 2 \mathrm{Fwww}$. humanas.unal.edu. co\%2Fliteratura $\% 2$ Findex.php $\% 2$ Fdownload_file $\% 2$ Fvie w\%2F111\&ei=KRObVbroIYe1ggStlor4CQ\&usg = AFQj CNEtVVaZ2lMCmB-s8yXcPEbhqvVcBA

VV.AA. (1987). La última cena. Poesía peruana actual. Lima: Asaltoalcielo/ Naylamp.

VV.AA. (1916). Las voces múltiples. Lima: Librería Francesa Científica E. Rosay.

WELLEK, R., Warren, A. (1966). Teoría literaria. Madrid: Gredos.

WILLIAMS, R. (2001). Cultura y sociedad. Buenos Aires: Nueva Visión. 